\title{
Terahertz Response of a Point Contact Based on CdTe/CdMgTe Quantum Well in Magnetic Field
}

\author{
I. Grigelionis ${ }^{a}$, M. Bialek ${ }^{a}$, M. Grynberg ${ }^{a}$, M. CzApkiewicz $^{b}$, V. Kolkovskiy $^{b}$,

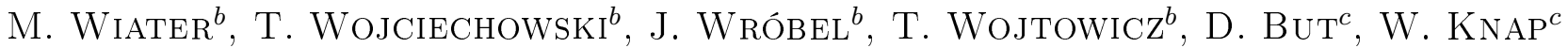 \\ AND J. ŁUSAKOWSKI ${ }^{a}$ \\ ${ }^{a}$ Institute of Experimental Physics, Faculty of Physics, University of Warsaw, Hoża 69, 00-681 Warsaw, Poland \\ ${ }^{b}$ Institute of Physics, PAS, al. Lotników 32/46, 02-668 Warsaw, Poland \\ ${ }^{c}$ TERALAB \& L2C CNRS, Université Montpellier 2, 34095 Montpellier, France
}

\begin{abstract}
A photoresponse at $\mathrm{THz}$ frequencies of a quantum point contact fabricated on a CdTe/CdMgTe quantum well was studied at low temperatures as a function of magnetic field. The spectra show a structure which was interpreted as resulting from the cyclotron resonance and magnetoplasmon excitations. The wavelength of the fundamental magnetoplasmon mode was found to be about $2 \mu \mathrm{m}$ which coincides with one of dimensions of the point contact. We also discuss the possibility of coupling of magnetoplasmon modes to shallow impurity transitions in the quantum well.
\end{abstract}

PACS: $85.30 . \mathrm{Hi}, 73.20 . \mathrm{Mf}$

\section{Introduction}

Quantum point contacts (QPCs) offer the possibility to investigate basic physical properties of matter, in particular in transport experiments [1-5]. Typically, in a QPC one can change the spatial extension of the current-conducting channel by polarizing lateral gates [1]. Such a configuration resembles that of a field-effect transistor (FET) with a split-gate electrode. It was shown that QPCs can be used as detectors of $\mathrm{THz}$ radiation; this subject, however, has not been intensively studied by now, and currently only a few reports can be found in the literature [6-8].

In the present paper, we study a $\mathrm{THz}$ response of a QPC fabricated on a CdTe/CdMgTe quantum well. The problem resembles that of a $\mathrm{THz}$ response of high electron mobility FETs (see, for example, Ref. [9]). An experience in investigation a $\mathrm{THz}$ response of FETs shows that one of the most important problems is that of coupling of the incident electromagnetic radiation to a two-dimensional electron gas (2DEG) in the transistor channel. In particular, this leads to the necessity of fabrication of special antennas which increase the efficiency of the coupling [10].

Generally, the problem of coupling of electromagnetic waves to a 2DEG electron gas is non-trivial, particularly in samples which show spatial features comparable to or smaller than the wavelength of the incident radiation. These complications result from the diffraction which generates (within the sample) a pattern of the electromagnetic field which is difficult to control. Let us assume that the magnetic field is perpendicular to the $2 \mathrm{DEG}$ plane, as it is in the present experiment. One can distinguish two basic experimental configurations: the Faraday geometry when the incident electromagnetic wave and the excited plasmon move in parallel to the magnetic field and the Voigt configuration when these directions are perpendicular. This discrimination is clear in the case of an infinite sample without any additional pattern on the surface. However, in reality, the samples are limited in size and additional metallization on the surface is often present which leads to diffraction of the incident waves. As a result, the wave which excites plasmons propagates within the sample parallel to the surface (the Voigt geometry) in spite of the fact that the incident wave is perpendicular to the surface (the Faraday geometry). This effect of diffraction has been used in the first investigation of plasmons in 2DEG [11] and ever since then in many experiments. A classical approach to plasma studies consists in investigation of a sample with a metallic grid deposited on the surface. The grid acts as a diffraction grating which generates waves propagating parallel to the surface. These waves carry an in-plane momentum acquired in the diffraction process which enables their coupling to plasmons in a 2DEG. Thus, plasmons in 2DEG should be described by formula corresponding to the Voigt geometry, in spite of the fact that the experimental configuration - from the point of view of the incident wave - is the Faraday geometry.

However, there are incident waves which are not scattered and they penetrate the sample in the direction of the magnetic field. These waves cannot excite plasmons but they can excite a single-particle cyclotron resonance (CR) transition. In the Faraday geometry considered here for a $2 \mathrm{DEG}$, this resonance is not coupled to a collective excitation of 2DEG and is given by the frequency $\omega_{\mathrm{c}}=e B / m^{*}$, where $e$ and $m^{*}$ are the electron charge and effective mass, respectively, and $B$ is the magnetic field. 
In the present experiment, the magnetic field is perpendicular to the 2DEG layer and the incident $\mathrm{THz}$ radiation is parallel to the magnetic field. Taking into account the above considerations, one should expect both the "bare" CR in the Faraday configuration and magnetoplasmon excitations in the Voigt configuration. In the case of plasma excitation in the Voigt geometry, the resonant response of the system occurs at the frequency [12]:

$$
\omega^{2}=\omega_{\mathrm{p} j}^{2}+\omega_{\mathrm{CR}}^{2},
$$

where $\omega_{\mathrm{p} j}$ is the frequency of $j$-th plasmon mode at zero magnetic field. Since the scattering of incident waves generates a series of harmonics of the fundamental mode, we expect that the wavevectors of the excited plasmons will be $k_{\mathrm{p} j}=j k_{\mathrm{p} 0}$, where $k_{\mathrm{p} 0}$ is a wave vector of the fundamental mode and $j$ is an integer.

The plasmon dispersion (i.e., a dependence of $\omega_{\mathrm{p} j}$ on the plasmon wave vector $k_{\mathrm{p} j}$ ) is given by [12]:

$$
\omega_{\mathrm{p} j}^{2}=\frac{n_{\mathrm{s}} e^{2} k_{\mathrm{p} j}}{2 \varepsilon \varepsilon_{0} m^{*}},
$$

where $n_{\mathrm{s}}$ is a $2 \mathrm{DEG}$ density and $\varepsilon$ - an effective dielectric constant of the material.

\section{Samples and experiment}

The sample investigated was a QPC formed on a $\mathrm{CdTe} / \mathrm{CdMgTe}$ single quantum well with the width of $15 \mathrm{~nm}$ grown on a semi-insulating GaAs substrate. The spacer thickness was $15 \mathrm{~nm}$ which was followed by a $5 \mathrm{~nm}$ thick layer doped with iodine donors. The $\mathrm{Mg}$ content in the barrier was $30 \%$. The electron sheet concentration $n_{\mathrm{s}}$ and the mobility $\mu$ at $1.5 \mathrm{~K}$ were $4.65 \times 10^{11} \mathrm{~cm}^{-2}$ and $8.8 \times 10^{5} \mathrm{~cm}^{2} /(\mathrm{V} \mathrm{s})$, respectively. The mesa of a $\mathrm{QPC}$ was formed by the electron beam litography. In Fig. 1a, a scanning electron microscopy picture of the sample is shown. The two mesas, facing one to the other with their wedge-shaped borders, are the gate contacts and the mesa between them is the channel. At the narrowest place, the channel width is $460 \mathrm{~nm}$. Electrical contacts to the sample were made by indium soldering.

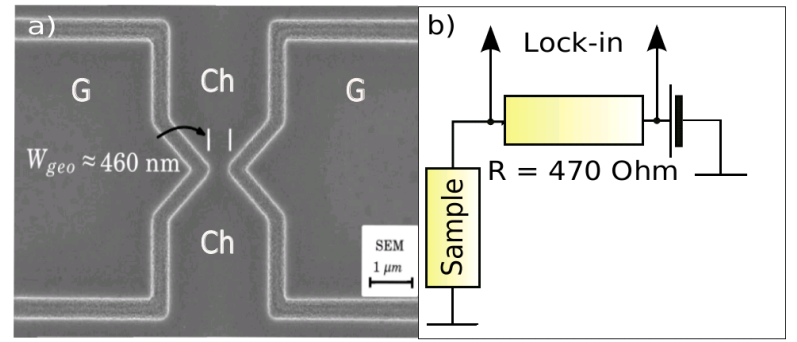

Fig. 1. (a) An STM picture of a QPC studied. Two opposite wedge-shaped mesas are the gates $(\mathrm{G})$ and the mesa with a "bottleneck" is the channel $(\mathrm{Ch})$.

(b) Scheme of electrical connections.

Photoconductivity of the QPC at $\mathrm{THz}$ frequencies was investigated as a function of the magnetic field. The sample was illuminated with a monochromatic $\mathrm{THz}$ radiation from an optically pumped molecular laser. Three different lines $(118.8 \mu \mathrm{m}, 163 \mu \mathrm{m}$ and $186 \mu \mathrm{m})$ were used for photocurrent excitation and the magnetic field was swept up to $10 \mathrm{~T}$. The QPC was biased from an external voltage source along the channel and the photocurrent was measured as a voltage drop on a load resistor connected in series with the sample (Fig. 1b). The signal was recovered with a lock-in technique with the chopping frequency of the $\mathrm{THz}$ laser as the reference. All measuremens were done at pumped liquid helium temperature of $T=1.8 \mathrm{~K}$.

\section{Results and discussion}

A spectrum of the photocurrent in magnetic field at $\mathrm{THz}$ wavelength of $118.8 \mu \mathrm{m}$ is shown in Fig. 2. No photoresponse was observed at magnetic fields between 0 and $7.5 \mathrm{~T}$ at this photon wavelength. In the case of the laser lines of $163 \mu \mathrm{m}$ and $186 \mu \mathrm{m}$, a non-zero photoresponse was observed also just only in a small range of $B$ below the CR transition. According to the considerations presented in Introduction, we interpret the peak at the highest magnetic field as the "bare" CR transitions and peaks at lower magnetic fields as subsequent plasmon resonances which correspond to plasmon wave vector $k_{\mathrm{p} j}$ with $j=1,2, \ldots$ A CR electron effective mass $m_{\mathrm{CR}}^{*}$ determined from the experimental data for three different laser lines is $0.1029 \pm 0.0002 m_{e}$. The spectra were deconvoluted into a series of Lorentzian peaks and the positions of the peaks in the magnetic field were used to calculate the values of $\omega_{\mathrm{p}}$ according to Eq. (1).

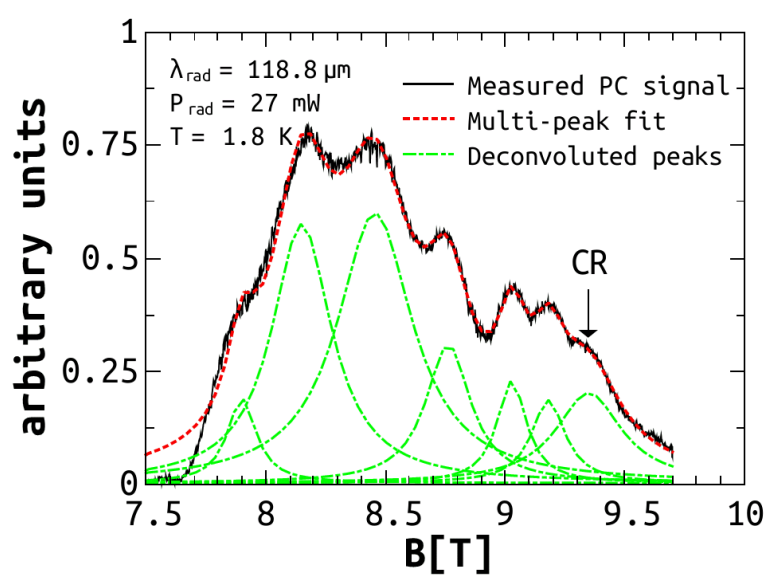

Fig. 2. Dependence of a photoresponse on magnetic field at $\mathrm{THz}$ wavelength $118.8 \mu \mathrm{m}$. Solid line represents the experimental data, dashed line - a photoresponse obtained from a multi-peak fitting, dashed-dotted line - peaks extracted from fitting.

Values of $\omega_{\mathrm{p} j}$, calculated from the peak position in the magnetic field are shown in Fig. 3 as a function of the plasmon mode number, $j$. This result indicates that the plasmon dispersion relation obeys a square root law, predicted by theory [12]. In order to find the value of the fundamental plasmonic resonance harmonics wave vec- 
tor $k_{\mathrm{p} 0}$, experimentally obtained plasmon dispersion relations were fitted with a square root function according to Eq. (2). Finally, from $k_{\mathrm{p} 0}$ value the plasmon wavelength $\lambda_{\mathrm{p} 0}$ was calculated (see the inset to Fig. 3). By analyzing dimensions of the QPC sample it was deduced that $\lambda_{\mathrm{p} 0}$ values corresponds to the sample channel width (see Fig. 1). This result means that a lateral spatial confinement determines the wave vector of the fundamental magnetoplasmon mode which gives a tool to design $\mathrm{THz}$ detectors based on excited plasmons.

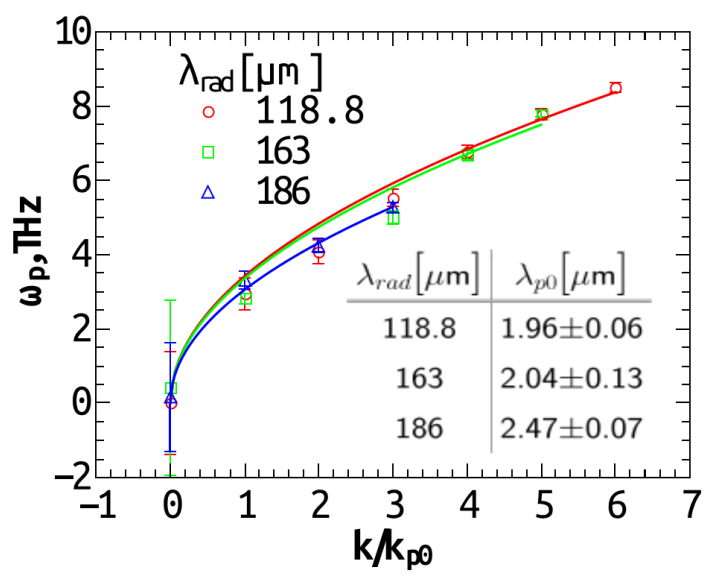

Fig. 3. Magnetoplasmon dispersion relations calculated from Eq. (1) for three laser lines (points). Solid lines denote fits of the square root dependence to plasmon dispersion relations. Inset: computed plasmon wavelengths $\lambda_{\mathrm{p} 0}$ for different laser lines.

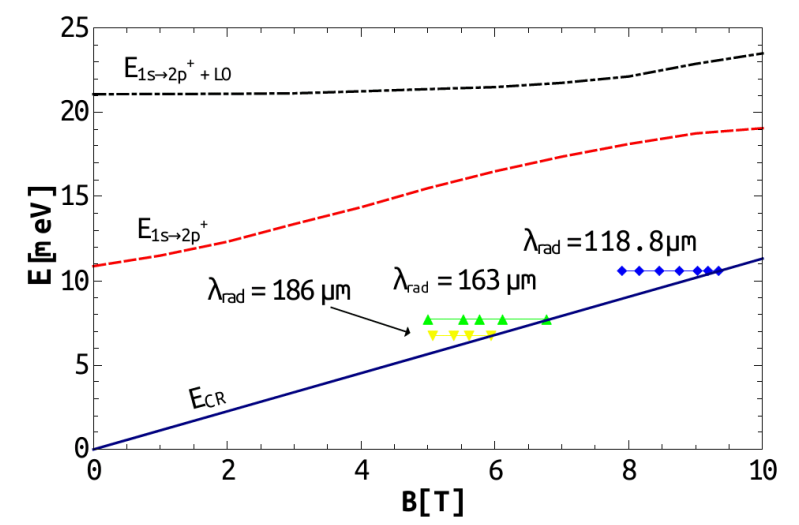

Fig. 4. Comparison between magnetoplasmon for different laser frequencies energies (symbols), cyclotron resonance energy (solid line), shallow donor impurity energy at bulk (dashed line) (adapted from [13]) and at interface of QW (dashed-dotted line).

To look for possible other excitations in the investigated structure, we compared the plasmon energies with energy of shallow donor impurities transitions in CdTe. The results of comparison are shown in Fig. 4.

Here the fundamental plasmonic resonance energies are depicted by symbols, the CR energy by a solid line, the shallow impurity transition energy in bulk $E_{1 s \rightarrow 2 p^{+}}$[13] by dashed lines and the $E_{1 s \rightarrow 2 p^{+}} / 4$ by dash-dotted lines. One must take into consideration that we are dealing with a quantum well, and the shallow donor binding energy strongly depends on the donor position in the well. In an infinitely deep and wide $\left(L_{\mathrm{QW}} \gg a_{\mathrm{B}}\right)$ well, the binding energy changes from four times the bulk value at the center of the well to a value four times smaller than the bulk value at the well/barrier interface [14]. It is evident that the energy of intra donor transition falls within the range of magnetoplasmon excitations. In the present experiment, however, we have not observed any signature of coupling of these two types of transitions, but such a possibility should be kept in mind in an analysis of experimental data.

\section{Conclusions}

In conclusion, we have investigated a photoresponse of a quantum point contact in a magnetic field at $\mathrm{THz}$ frequency. The results were interpreted within a model of the cyclotron resonance and magnetoplasmon excitations. We showed that the wavelength of the plasmon fundamental mode was determined by dimensions of lithographically designed pattern on the sample surface.

\section{Acknowledgments}

This work was partially supported by a Polish Ministry of Higher Education project NN515071937, the EU within European Regional Development Found, through grant Innovate Economy (POIG.01.01.02-00-008/08) and by $\mathrm{N} 202 / 103936$ project.

\section{References}

[1] B.J. Wees, H. van Houten, C.W.J. Beenakker, J.G. Williamson, L.P. Kouwenhoven, D. van d. Marel, C.T. Foxon, Phys. Rev. Lett. 60, 848 (1988).

[2] S. Datta, Electronic Transport in Mesoscopic Systems, Cambridge Studies in Semiconductor Physics and Microelectronic Engineering, Cambridge University Press, 1997.

[3] B.J. Wees, L.P. Kouwenhoven, H. Houten, van; C.W.J. Beenakker, J.E. Mooij, C.T. Foxon, J.J. Harris, Phys. Rev. B 38, 3625 (1988).

[4] A.B. Fowler, A. Hartstein, R.A. Webb, Phys. Rev. Lett. 48, 196 (1982).

[5] T.J. Thornton, M. Pepper, H. Ahmed, D. Andrews, G.J. Davies, Phys. Rev. Lett. 56, 1198 (1986).

[6] T.J.B.M. Janssen, J. Singleton, N.K. Patel, M. Pepper, J.E.F. Frost, D.A.R.J.C. Maan, M.J.C. Maan, G.A.C. Jones, J. Phys., Condens. Matter 6, L163 (1994).

[7] R.J. Heron, R.A. Lewis, B.E. Kane, G.R. Facer, R.G. Clark, A.S. Dzurak, N.E. Lumpkin, R.P. Starrett, D.G. Rickel, L.N. Pfeiffer, K.W. West, Appl. Phys. Lett. 75, 3150 (1999). 
[8] J.W. Song, N.A. Kabir, Y. Kawano, K. Ishibashi, G.R. Aizin, L. Mourokh, J.L. Reno, A.G. Markelz, J.P. Bird, Appl. Phys. Lett. 92, 223115 (2008).

[9] J. Łusakowski, Acta Phys. Pol. 119, 114 (2011).

[10] P. Kopyt, J. Marczewski, K. Kucharski, J. Łusakowski, W. Gwarek, in: Proc. 36th Conf. on Infrared, Millimeter and Terahertz Waves, Houston, 2011.

[11] S.J. Allen, D.C. Tsui, R.A. Logan, Phys. Rev. Lett. 38, 980 (1977).
[12] T. Ando, A.B. Fowler, F. Stern, Rev. Mod. Phys. 54, 437 (1982).

[13] M. Grynberg, S. Huant, G. Martinez, J. Kossut, T. Wojtowicz, G. Karczewski, J.M. Shi, F.M. Peeters, J.T. Devreese, Phys. Rev. B 54, 1467 (1996).

[14] J.D. Levine, Phys. Rev. 140, A586 (1965). 